\title{
SOP Abklärung bei Verdacht auf Suizidalität
}

Barbara Schneider, Manfred Wolfersdorf

\begin{abstract}
Die Abklärung von Suizidalität ist bei der psychiatrischen Erstuntersuchung immer erforderlich. Suizidalität ist Ausdruck einer psychiatrischen Notfallsituation, die ein rasches Handeln und eine weitere medizinisch-psychiatrisch-psychotherapeutische Versorgung erfordert. Ein standardisiertes Vorgehen zur Abklärung der Suizidalität ist hilfreich.
\end{abstract}

\section{Einleitung}

Erst durch die Zuordnung zur Psychiatrie im 19. Jahrhundert wurde die Grundlage für das heutige „medizinischpsychosoziale Paradigma“ von Suizidalität geschaffen und damit die Jahrhunderte lang bestehende religiösphilosophischen Sichtweise abgelöst. Suizidalität ist ein ernsthaftes Gesundheitsproblem, dessen Einschätzung nach dem eigenen Urteil des Untersuchers auf der Information durch den Betroffenen und dem fachlichen Wissen um Suizidalität fördernde Risikofaktoren und Gefährdungszeichen beruht [1]. Sichere Testverfahren, z. B. Biomarker, zur Abklärung bei Verdacht auf Suizidalität gibt es nicht.

\section{Epidemiologie}

Weltweit versterben jährlich mehr als 800000 Menschen durch Suizid (Weltgesundheitsorganisation 2014). Die Suizidrate pro 100000 der Bevölkerung beträgt weltweit 11,4 (Männer 15,0; Frauen 8,0). In den letzten 45 Jahren haben die Suizidraten weltweit um $60 \%$ zugenommen. Von allen Suiziden ereigneten sich $75 \%$ in Ländern mit niedrigem und mittlerem Einkommen, woraus die WHO einen Zusammenhang zwischen psychosozialer Lebenssituation und Suizidalität ableitet. Suizid ist eine der drei häufigsten Todesursachen in den Altersgruppen zwischen 15 und 44 Jahren in einigen Ländern und die zweithäufigste Todesursache in der Altersgruppe zwischen 10 und 24 Jahren. Die Suizidraten waren weltweit am höchsten bei Männern und Frauen bei den 70-Jährigen und Älteren. Die häufigsten Suizidmethoden waren Intoxikation durch Pestizide, Erhängen und Erschießen. Fast $80 \%$ aller Suizidopfer sind Männer. Für diese Verteilung zwischen den Geschlechtern gibt es viele mögliche Gründe: Unterschiede im Hilfesuchverhalten, Präferenzen für bestimmte Suizidmethoden, aber auch Verfügbarkeit und das Muster des Alkoholkonsums. Suizid führte nach Schätzungen zu 1,3\% der „total global burden of disease“
(Krankheitslast, gemessen in Disability-Adjusted Life Years, DALY) und wird im Jahr 2020 auf 2,4\% in Ländern mit Marktwirtschaft und in früheren sozialistischen Staaten ansteigen (Weltgesundheitsorganisation 2014).

In Deutschland ist in den letzten 30 Jahren die Zahl der Suizide zurückgegangen: In Gesamtdeutschland halbierte sich die Zahl der Suizide nahezu. 1982 ereigneten sich noch 18451 Suizide; im Jahr 2015 lag die Zahl aller Suizide bei 10080 (7398 Männer und 2682 Frauen). Suizidprävention einschließlich gesellschaftlicher Veränderungen spielt langfristig für die Entwicklung von Suizidraten eine große Rolle. Männer haben in Deutschland wie in vielen anderen Industriestaaten eine fast dreimal höhere Suizidrate als Frauen [2]. In Deutschland nehmen mit zunehmendem Lebensalter die Suizidraten zu. Während im Jahr 2015 bei den 20- bis 25-Jährigen die Suizidrate lediglich 6,9 pro 100000 Einwohner betrug, belief sie sich in der Altersgruppe der 85 - bis 89-Jährigen auf 33,0 pro 100000 (Statistisches Bundesamt Deutschland 2017). Die häufigsten Suizidmethoden in Deutschland sind Erhängen, Vergiften und der Sturz aus der Höhe. Es muss davon ausgegangen werden, dass sich etwa zehn- bis vierzigmal so viele Suizidversuche wie vollendete Suizide ereignen.

\section{Definition von Suizidalität}

Suizidalität ist ein zutiefst menschliches Geschehen und Erleben, das in seiner Komplexität nie vollständig verstehbar sein wird. Der Bereich, in welchem uns Suizidalität überwiegend begegnet, ist das medizinisch-psychosoziale Umfeld von Beratung, Krisenintervention und medizinisch-psychotherapeutischer bzw. -psychiatrischer (Notfall-)Behandlung [3]. Auch mit dieser Einschränkung wurde eine Vielzahl von Definitionen zusammengetragen (z. B. [4]). Letztlich gibt es keine Definition von Suizidalität, die das gesamte Spektrum dieses Phänomens abdeckt. Heute ist die Definition der WHO am geläufigsten: 
Unter Suizidalität versteht man eine Reihe von Denk- und Verhaltensweisen, die Suizidgedanken oder -absichten, Suizidpläne, Suizidversuche und Suizide einschließen (WHO). Eine suizidale Handlung als solche zu benennen, liegt beim Handelnden [3]. Die Deutung einer Handlung als Ausdruck von Suizidalität liegt beim Beobachter; dabei gehen dessen Fachwissen wie auch der Eindruck auf therapeutischer Seite bzw. das Übertragungsgefühl mit ein.

In den meisten Fällen entwickelt sich Suizidalität rasch: Die Bedenkzeit von der Suizididee bis zur Durchführung eines Suizidversuchs liegt bei $2 / 3$ der Betroffenen unter 24 Stunden und die Zeitspanne vom Entschluss zur Handlung bis zur Durchführung eines Suizidversuchs liegt in $97 \%$ aller Fälle unter 24 Stunden, in 58\% sogar unter einer Stunde [5]. Letztlich bedeutet dies, dass Suizidalität in der Regel eine temporäre Erscheinung ist und eine hohe zeitliche Dynamik hat.

Pöldinger hat 1968 die „Stadien suizidaler Entwicklungen“ beschrieben: nach einem Erwägungsstadium mit Suizidideen und erhaltener Distanzierungsfähigkeit folgt das Ambivalenzstadium mit Hin- und Herschwanken zwischen eigentlich „Nichtsterbenwollen“, aber so Nichtweiterlebenkönnen und daraus entspringenden Appellen und Suizidankündigungen; am Ende steht das Entschlussstadium mit aufgehobener Distanzierungsfähigkeit und Steuerungsfähigkeit, mit Resignation und letztendlich trügerischer Ruhe durch den erfolgten Entschluss der Selbsttötung [6].

Das Kontinuitätsmodell von Suizidalität nach Wolfersdorf (2008) [7] beschreibt verschiedene Gefährdungsgrade mit unterschiedlich hohem Handlungsdruck und sich daraus ergebenden Konsequenzen für fürsorgliche Maßnahmen der Fremdverantwortung.

- Wunsch nach Ruhe, Pause, Unterbrechung im Leben ohne Intention zu versterben.

- Todeswunsch: aktuell oder in der Zukunft, ohne aktive Handlung und ohne Handlungsdruck.

- Suizidideen oder sich zwanghaft aufdrängende Suizidgedanken: mehr oder weniger konkret als mögliche Handlungsweise gedacht; häufig Ausdruck von Ambivalenz; ohne konkreten Handlungsdruck; eher passiv.

- Suizidabsichten: Suizidideen mit konkreter Planung und Absichtserklärung zur Durchführung; deutlicher als Drang erlebter Handlungsdruck.

- Suizidhandlung: vorbereiteter Suizidversuch, begonnen und abgebrochen (unter Fremd- und Selbsteinfluss), gezielt geplant, impulshaft durchgeführt.

Eine parasuizidale Handlung sieht wie eine suizidale Handlung aus. Die Selbstdestruktion hat jedoch kaum Todesintention, wenngleich die Gefahr des Sterbens in Kauf genommen wird. Die Handlung hat oft ausgeprägten ap- pellativen oder instrumentellen Charakter: etwas soll erreicht werden oder es wird auf eine Veränderung abgezielt.

\section{Risikofaktoren und Warnzeichen}

Verschiedene Autoren und Organisationen, die im Bereich der Suizidprävention aktiv sind, haben unterschiedliche Warnzeichen und Risikofaktoren aufgeführt [8]. Bekannte Risikofaktoren sind vor allem psychische Erkrankungen, insbesondere Depressionen, Suchterkrankungen, Persönlichkeitsstörungen, schizophrene Psychosen, körperliche Erkrankungen, akute belastende Lebensereignisse, frühere Suizidversuche und Suizidversuche in der Familienanamnese. Zu den Warnzeichen gehören unter anderem das Äußern von Suizidgedanken, Sinnlosigkeit im Leben, das Gefühl, für andere eine Last zu sein, sozialer Rückzug, Verabschieden bei anderen, das Verschenken von wertvollen Besitztümern, Angst, Erniedrigung, Hoffnungslosigkeit und Verzweiflung, ausgeprägte Stimmungsschwankungen.

Die Information zu Risikofaktoren [9] sollte man grundsätzlich bei der Risikoabschätzung berücksichtigen. Dies ist auch dann zu bedenken, wenn in der aktuellen Situation keine konkreten Suizidgedanken oder -absichten eruierbar sind. In psychiatrischen Kliniken ereignen sich in der ersten Zeit nach der stationären Aufnahme die meisten Suizide [10].

\section{Grundprinzipien der Krisenintervention - was ist im Gespräch mit Suizidalen zu beachten}

In der Krisenintervention geht es um die Herstellung einer Beziehung, die hilfreichen, stützenden und damit per se bereits präventiven Charakter hat, um die Diagnostik von Suizidalität und die Risikoeinschätzung sowie die Diagnostik von psychischer Störung, um das Management der aktuellen Situation sowie um die Therapieplanung einer zugrunde liegenden psychischen Erkrankung bzw. einer emotionalen Krise oder Belastungsoder Anpassungsreaktion.

Wesentliche Voraussetzung für ein erfolgreiches Management von Suizidalität ist es, sie als Signal seelischer Not des Betroffenen anzusehen, als Ausdruck dessen, dass ein Mensch in Not ist, der Hilfe, Zuwendung und Verstehen benötigt. Dies soll auch mit dem positiven Stellenwert des Notsignals für die Person zu erkennen sein, die letztendlich nicht anders handeln konnte und zur Beendigung einer sehr quälend erlebten Befindlichkeit diesen subjektiv bedeutsamen Weg gewählt hat. Basis ist eine gute, auch Belastung aushaltende, offene und vertrauensvolle Beziehung, in der Schutz und Fürsorge, Stüt- 
zung, emotionale Entlastung, Vermittlung von Krankheits- bzw. Krisenkonzept unter Beachtung der aktuellen Psychopathologie sowie der aktuellen Psychodynamik deutlich werden [11].

\section{Wann ist eine Abklärung der Suizidalität erforderlich?}

Eine Abklärung der Suizidalität ist immer im Rahmen einer psychiatrischen Erstuntersuchung bei jedem Patienten erforderlich. Außerdem muss bei jeder neu aufgetretenen Krisensituation, vor Entlassung, aber auch

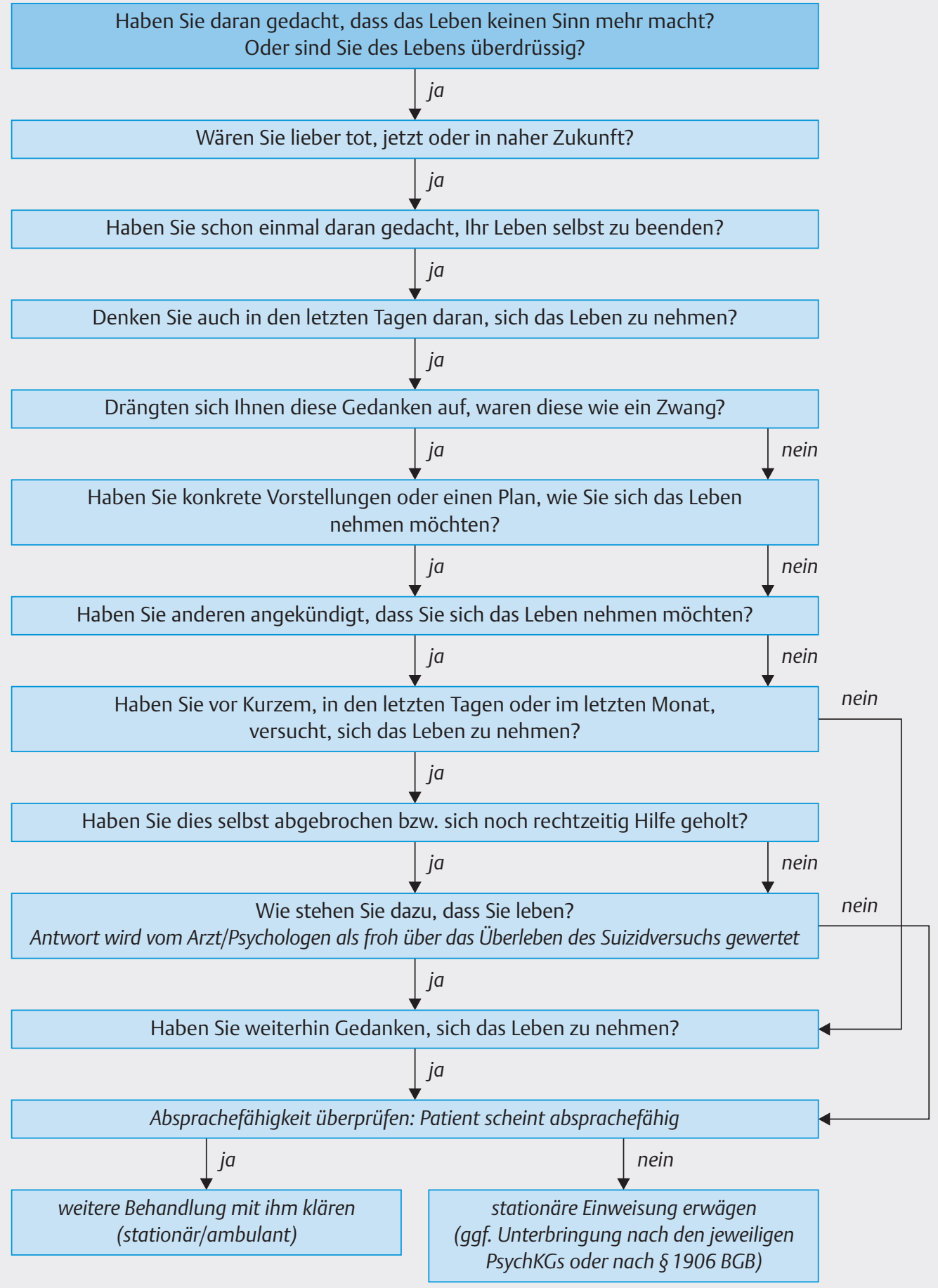

- Abb. 1 Entscheidungsbaum und weiteres Vorgehen. 
bei jeder plötzlich auftretenden (scheinbaren) Besserung, bei fremdanamnestischen Hinweisen sowie bei Suizidalität fördernder Symptomatik und bei Verschlechterung erneut nach Suizidalität gefragt werden ( $\bullet$ Abb.1). Grundsätzlich kann in jedem Stadium des Behandlungsverlaufs Suizidalität neu auftreten. Daher ist eine nach den Besonderheiten des Einzelfalls ausgerichtete, regelmäßige Erfassung notwendig. Es muss immer eine Dokumentation des erhobenen Befundes erfolgen. Formulierungen müssen in der Dokumentation immer klar und eindeutig sein.

\section{Interessenkonflikt}

Die Autoren geben an, dass keine Interessenkonflikte vorliegen.

\section{Autorinnen/Autoren}

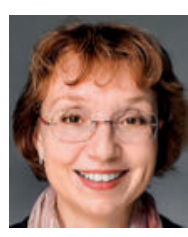

\section{Barbara Schneider}

Prof. Dr. med. Barbara Schneider ist Chefärztin der Klinik für Abhängigkeitserkrankungen und Allgemeinpsychiatrie der LVR-Klinik Köln und Vorsitzende der Deutschen Gesellschaft für Suizidprävention (DGS) und des Nationalen Suizidpräventionsprogramms für Deutschland (NaSPro)

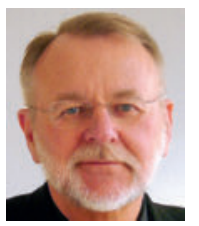

\section{Manfred Wolfersdorf}

Prof. Dr. med. Dr. h.c. Manfred Wolfersdorf ist ehemaliger ärztlicher Direktor und Chefarzt des Bezirkskrankenhauses Bayreuth, akademisches Lehrkrankenhaus der Universität ErlangenNürnberg, sowie Gründer und ehemaliger Leiter des Referat Suizidologie der DGPPN.

\section{Korrespondenzadresse}

Prof. Dr. med. Barbara Schneider

LVR-Klinik Köln

Abteilung für Abhängigkeitserkrankungen

und Allgemeinpsychiatrie

Wilhelm-Griesinger-Str. 23

51109 Köln

b.schneider@lvr.de

\section{Literatur}

[1] Althaus D, Hegerl U. Ursachen, Diagnose und Therapie von Suizidalität. Nervenarzt 2004; 75: 1123-1134; quiz 1135

[2] Statistisches Bundesamt Deutschland. Sterbefälle nach äußeren Ursachen und ihren Folgen (ab 1998). Wiesbaden: Statistisches Bundesamt; 2018. Im Internet: http://www.gbe-bund. de/oowa921-install/servlet/oowa/aw92/dboowasys921. xwdevkit/xwd_init?gbe.isgbetol/xs_start_neu/\&p_aid= 3\&p_aid $=57548728 \&$ nummer $=562 \&$ p_sprache $=$ D\&p_indsp= -\&p_aid=40742185; Stand: 30.09 .2018

[3] Wolfersdorf M. Depression und Suizid. Bundesgesundheitsbl Gesundheitsforsch Gesundheitsschutz 2008; 51: 443-450

[4] Bronisch T. Der Suizid. Ursachen, Warnsignale, Prävention. 6. Aufl. München: C.H. Beck; 2014

[5] Linden KJ. Der Suizidversuch. Versuch einer Situationsanalyse. Stuttgart: Enke; 1969

[6] Pöldinger W. Die Abschätzung der Suizidalität. Bern, Stuttgart, Wien: Hans Huber Verlag; 1968

[7] Wolfersdorf M. Suizidalität. Nervenarzt 2008; 79: 1319-1334

[8] Franklin JC, Ribeiro JD, Fox KR et al. Risk factors for suicidal thoughts and behaviors: A meta-analysis of 50 years of research. Psychol Bull 2017; 143: 187-232

[9] Schneider B. Risikofaktoren für Suizid. Regensburg: Roderer; 2003

[10] Schneider B, Wolfersdorf M, Wurst FM. Suizid und Suizidprävention im psychiatrischen Krankenhaus. Psych up2date 2017; 5: $445-463$

[11] Wolfersdorf M, Mauerer C, Franke C et al. Krisenintervention bei Suizidalität im ambulanten und stationären psychiatrischpsychotherapeutischen Bereich. Psychotherapie 1999; 4: 156-164

Bibliografie

DOI https://doi.org/10.1055/a-0630-8696

PSYCH up2date 2018; 12: 439-442

(c) Georg Thieme Verlag KG Stuttgart · New York ISSN 2194-8895 